\title{
Educación Especial y sus implicancias en contextos de diversidad cultural: análisis desde La Araucanía ${ }^{1}$
}

\author{
Special needs education and its implication in cultural diversity contexts: \\ analysis from La Araucanía
}

\section{Educação Especial e suas implicações em contextos de diversidade cultural: uma análise a partir da Aracucanía}

Recepción: 30/09/2019

Evaluación: 30/09/2019

Aceptación: 01/04/2020

Artículo de Investigación - Revisión

https://doi.org/10.19053/01227238.10105

Ximena Gutiérrez-Saldivia ${ }^{2}$

https://orcid.org/0000-0003-1258-1562

Catalina Rivera Gutiérrez ${ }^{3}$

https://orcid.org/0000-0001-9326-7051

Universidad Católica de Temuco, Chile

\section{RESUMEN}

Objetivo: Este artículo plantea cultural podría constituir un dispositivo un análisis crítico sobre la Educación de control para homogeneizar a la poblaEspecial en contextos de diversidad cultural confrontando supuestos teóricos asociados al enfoque médico, la teoría del etiquetamiento y perspectiva de poder. Método: El análisis se desarrolla a través de la revisión de literatura científica y normativa nacional e internacional, empleando un método documental-descriptivo. La recuperación de documentos se realizó desde las bases de datos Web of Science, Scopus y Scielo. Estrategias: Un análisis de los documentos recuperados ha permitido establecer que la Educación Especial en contextos de diversidad ción escolar, lo que se realiza a través de un sistema de clasificación y etiquetado, donde se utilizan procedimientos como el examen y el diagnóstico, en base a estándares aplicados por el grupo dominante. Originalidad: El artículo contribuye a la problematización de la educación especial en contextos educativos interculturales para avanzar hacia respuestas educativas culturalmente pertinentes. Se puede concluir que, tanto en Latinoamérica como en la región de La Araucanía se requiere repensar la Educación Especial desde nuevos marcos epistemológicos

$1 \quad$ Agradecimientos a ANID-BECA de Doctorado Nacional n. 21170975.

2 Máster en Intervención Psicopedagógica. Estudiante de Doctorado en Educación en Consorcio, Universidad Católica de Temuco (UCT). Profesora de planta temporal en la carrera de Pedagogía en Educación Diferencial, Facultad de Educación, Departamento de Infancia y Educación Básica, UCT, Chile, correo electrónico: xgutierrez.saldivia@gmail.com

3 Doctora en Educación por la Universidad Católica de Temuco, Magíster en Educación Física por la Universidad de la Frontera, Facultad de Educación, Universidad Católica de Temuco, correo electrónico: cata.riverag@gmail.com 
-como la interculturalidad crítica- que permitan reconocer y valorar la diversidad cultural.
Palabras clave: educación especial, normalidad, necesidades educativas especiales, teoría del etiquetado.

\section{ABSTRACT}

This work aims at analyzing the special needs education through an epistemological reflection in contexts of social and cultural diversity. The analysis is developed, through the review of scientific literature in national and international regulations, using a documentary-descriptive method. The analysis considers the theory of abnormality that underlies the medical model applied to education, the theory of labeling and the perspective of power. The results reveal that Special Needs Education could make up a regulatory control to homogenize the school population, which is materia- lized through a classification and labeling system through examination and diagnostic procedures based on standards applied by the dominant group. It is concluded that, in order to move towards a possible conceptualization of Special Needs Education in indigenous territories such as La Araucanía, a new way of thinking is needed on issues related to special educational needs from a critical perspective in order to break western structure and canons.

Key words: Special Needs Education; normalcy; theory of labeling.

\section{RESUMO}

O objetivo deste trabalho é analisar a Educação Especial por meio de uma crítica epistemológica em contextos de diversidade social e cultural. A análise se desenvolve através da revisão da literatura científica e normativa nacional e internacional, aplicando um método documental-descritivo. A análise considera a teoria da anormalidade que subjaz no modelo médico aplicado à educação, a teoria da rotulação e a perspectiva do poder. Os resultados revelam que a Educação Especial poderia constituir um dispositivo de controle para homogeneizar a população escolar, que se materializa mediante um sistema de classificação e rotulação através de procedimentos de exames e diagnósticos com base em normas aplicadas pelo grupo dominante. Conclui-se que, para avançar para uma possível conceitualização da Educação Especial em territórios indígenas como La Araucanía, é necessária uma nova forma de pensar as questões associadas às necessidades educacionais especiais a partir de uma perspectiva crítica com o fim de romper com a estrutura e os cânones ocidentais.

Palavras chave: Educação especial; normalidade; necessidades educativas especiais; teoria da rotulação.

\section{INTRODUCCIÓN}

Una particularidad innegable de las sociedades es que en ellas coexisten diferentes grupos sociales y culturales, es decir, que su carácter es multicultural. Este fenómeno ha sido analizado en la actualidad principalmente desde los 
movimientos migratorios ${ }^{4}$. Autores como Besalú ${ }^{5}$ reconocen que las sociedades siempre han sido multiculturales, es decir, plurales y diversas desde diferentes puntos de vista ${ }^{6}$. Es importante considerar lo anterior ya que la diversidad social y cultural en Latinoamérica es una característica presente en su historia, que ha sido negada, invisibilizada, combatida y censurada, en nombre del orden y la unidad nacional, deseada por los Estados-Nación ${ }^{7}$.

Sobre lo anterior, la educación desempeña un papel clave en la dinámica de las sociedades, y es considerada como una actividad social necesaria para la transmisión de la cultura a las nuevas generaciones ${ }^{8}$. Desde este punto de vista "[...] sabemos que ésta [la educación] no es sino un instrumento en manos de la sociedad, que busca en esta práctica el cultivo de la savia que ha de conservarla y regenerarla, la transmisión de la cultura que haga posible su continuidad histórica" ${ }^{\prime \prime}$. Mientras que etimológicamente es definida como "la acción y efecto de educar, [que] procede del latín educare que significa conducir, guiar, orientar, etc. Hace, pues, referencia a un proceso asistido y dirigido hacia algún fin"10.

Cabe destacar que, desde una perspectiva sociológica integradora, la educación es entendida como "el conjunto de instituciones, personas, creencias, contenidos y prácticas tendientes a la socialización, transmisión cultural y formación diferenciada de las nuevas generaciones" ${ }^{\prime 11}$. Desde este punto de vista, subyace en los discursos políticos y reformas educativas el enfoque funcionalista de la educación y sociedad. El énfasis del enfoque funcionalista "es la estabilidad como condición normal de la sociedad, en la integración social y en la eficiencia del sistema social como un todo"12; dicha perspectiva posee el supuesto de la universalidad de la naturaleza humana. En palabras de Gimeno ${ }^{13}$, la educación basada en el principio universalista pretende la creación de comunidades homogéneas, que compartan hábito, valores y pensamientos. Así, la educación ha ocupado un lugar clave para lograr el control social y la homogeneización de la población, negando e invisibilizando la diversidad social y cultural, como es el caso latinoamericano, y en particular de los pueblos indígenas.

Desde el enfoque funcionalista, la sociedad, y específicamente en la educación, se ha construido una concepción de la diversidad como un aspecto negativo que debe ser clasificado, atendido y corregido, creando un imaginario del

4 Miquel Àngel Essomba, Liderar escuelas interculturales e inclusivas. Equipos directivos y profesorado ante la diversidad cultural y la inmigración (Barcelona: Graó, 2006).

5 Xavier Besalú, Diversidad cultural y educación (Madrid: Síntesis Educación, 2002).

6 Abraham Magendzo, “Alteridad y diversidad: componentes para la educación social”, Pensamiento Educativo vol. 37 (2005).

7 Francisco Fernández, "El estudio sociológico de la educación”, en Sociología de la Educación, coord. Francisco Fernández (Madrid: Pearson Prentice Hall, 2003).

8 Besalú, Diversidad cultural.

9 Ibíd., 33

10 Antonio Guerrero, Enseñanza y sociedad. El conocimiento sociológico de la educación (Madrid: Siglo XXI, 2005).

11 Ibíd., 16.

12 Carlos Ornelas, "Educación y sociedad: ¿Consenso o conflicto?", en Sociología de la educación. Corrientes contemporáneas, eds. Carlos Alberto Torres y Guillermo González Rivera (Buenos Aires: Miño y Dávila editores, 1994), 61.

13 José Gimeno Sacristán, “La construcción del discurso acerca de la diversidad y sus prácticas”, en Atención a la diversidad (Barcelona: Graó, 2000). 
otro como un ser diferente. Esta visión perpetuadora de la diversidad social y cultural se ha construido desde diferentes modalidades de contacto, como son la asimilación, segregación, marginación e integración, las que han sido utilizadas desde el grupo mayoritario hacia los grupos sociales y culturales minoritarios ${ }^{14}$. Ejemplo de esto es el sistema escolar, que a través de la institucionalización formal que imparte la escuela ha creado un espacio uniforme y asimilacionista que enseña una cultura común en su lenguaje, identidad, valores y pautas de conducta $^{15}$. En este sentido, la escuela transmite una única cultura, asumiendo que las otras son aproximaciones desviadas o insuficientes ${ }^{16}$. Esta afirmación, desde los procesos de colonización de los pueblos indígenas como el mapuche, ha creado imaginarios peyorativos, ya que en el pasado fueron considerados erróneamente como barbarie que debía ser civilizada ${ }^{17}$.

Por lo descrito, y desde la necesidad de atender y comprender a los estudiantes, el sistema escolar en general ha promovido estrategias compensatorias a través de programas educativos para grupos específicos, como aquellos que presentan Necesidades Educativas Especiales (en adelante NEE). En Chile, por ejemplo, el Ministerio de Educación (MINEDUC) propone como estrategia los Programas de Integración Escolar (en adelante PIE), desde la modalidad de Educación Especial. La Educación Especial chilena es entendida como una "modalidad del sistema educativo que desarrolla su acción de manera transversal en los distintos niveles, tanto en los establecimientos de educación regular como especial"18 $^{\prime 18}$ existiendo desde el año 1990 normativas de integración escolar ${ }^{19}$. Cabe destacar que la integración escolar se materializa en los establecimientos de educación regular a través de los PIE, que tienen como finalidad entregar apoyos educativos adicionales a estudiantes que presentan NEE con el propósito de contribuir al mejoramiento de los aprendizajes escolares ${ }^{20}$. Para hacer efectivos dichos apoyos, el MINEDUC "exige a las escuelas identificar a aquellos estudiantes con NEE, mediante un proceso de evaluación diagnóstica, que se traduce en la asignación de categorías de discapacidad o trastornos" ${ }^{\prime 21}$. Esto ocurre porque el modelo educativo chileno se basa en un sistema de subvención consistente en un subsidio económico que entrega el Estado a las escuelas por cada estudiante matriculado ${ }^{22}$. En el caso de las escuelas que cuentan con PIE,

\footnotetext{
14 Besalú, Diversidad cultural.

15 Fernández, "El estudio sociológico".

16 Ibíd.

17 Andrés Donoso, Educación y nación al sur de la frontera. Organizaciones mapuche en el umbral de nuestra contemporaneidad, 18801930 (Santiago de Chile: Pehuén Editores S.A., 2012).

18 Ministerio de Educación (MINEDUC), Ley Núm. 20.370 que establece la Ley General de Educación (Biblioteca del Congreso Nacional de Chile, 12 de septiembre de 2009).

19 M. ${ }^{a}$ Paulina Godoy, M. ${ }^{a}$ Luisa Meza y Alida Salazar, Antecedentes históricos, presente y futuro de la Educación Especial en Chile (Santiago de Chile: MINEDUC, 2004).

20 MINEDUC, Orientaciones técnicas para Programas de Integración Escolar (PIE) (Santiago de Chile: Gobierno de Chile, 2013).

21 Ximena Gutiérrez-Saldivia, Segundo Quintriqueo Millán y Vanessa Valdebenito Zambrano, "Carácter monocultural de la evaluación diagnóstica de necesidades educativas especiales en contexto mapuche”, Educaçao e Pesquisa vol. 45, (2019): 3. doi: http:// dx.doi.org/10.1590/S1678-4634201945200049.

22 Jesús Redondo, “El experimento chileno en educación: ¿Conduce a mayor equidad y calidad en la educación?”, Última Década vol.
} 
estas aumentan sus ingresos ya que se les paga una subvención adicional por cada estudiante diagnosticado con discapacidades o trastornos. Dichas políticas se relacionan con el modelo neoliberal y de subsidio que mantiene la educación escolar chilena ${ }^{23}$.

En contextos de diversidad social y cultural, quienes han sido más afectados por este tipo de políticas que fomentan el diagnóstico en el sistema escolar son los estudiantes indígenas, como representantes de una cultura minoritaria involuntaria, y estudiantes migrantes, como representantes de una cultura minoritaria voluntaria $^{24}$. Dichos estudiantes se encuentran excesivamente representados - en inglés se define con el sustantivo overrepresentation - en programas de Educación Especial ${ }^{25}$. En el caso de Chile se observa un aumento de estudiantes con NEE en escuelas de educación primaria que cuentan con PIE, existiendo en el 2004 una matrícula de 20746 estudiantes diagnosticados ${ }^{26}$, mientras que en el 2013 fueron $171712^{27}$ los estudiantes diagnosticados, aumentando ocho veces la matrícula de estudiantes en esta categoría.

Desde lo expuesto en los párrafos anteriores, y desde la normativa vigente en Chile, este trabajo se propone reflexionar críticamente sobre la Educación Especial en contextos de diversidad cultural, desde la región de La Araucanía y sus particularidades territoriales y culturales. Es importante abordarlo desde esta región ya que se caracteriza por su diversidad social y cultural. La Araucanía se destaca en el país por ser una de las regiones con mayor población rural e indígena mapuche del país, con un $29,1 \%$ y 34,3\%, respectivamente ${ }^{28}$. A su vez, la población que se reconoce como mapuche representa el mayor porcentaje estadístico $(79,8 \%)$ entre quienes se reconocen como indígenas dentro del territorio nacional. $\mathrm{O}$ sea, la población mapuche en el ámbito nacional es la que posee mayor representación, al igual que en La Araucanía, haciendo que su reconocimiento sea de importancia ${ }^{29}$. Por otra parte, las regiones del Biobío y La Araucanía son actualmente zonas que se mantienen bajo serios conflictos

13, n. ${ }^{\circ} 22$ (2005); María Oliva, "Política educativa chilena 1965-2009. ¿Qué oculta esa trama?," Revista Brasileira de Educação vol. $15,{ }^{\circ} 44(2010)$.

23 Jenny Assaél, Natalia Albornoz y Miguel Caro, "Estandarización educativa en Chile: tensiones y consecuencias para el trabajo docente", Educação Unisinos vol. 22, n. ${ }^{\circ} 1$ (2018). doi:https://doi.org/10.4013/edu.2018.221.09.

24 John Ogbu, "Cultural problems in minority education: Their interpretations and consequences-Part one: Theoretical backgroud", The Urban Review vol. 27, n. ${ }^{\circ} 3$ (1995).

25 Suzanne Donovan y Christopher Cross, eds., Minority Students in Special and Gifted Education (Washington, D.C.: The National Academies Press, 2002); Daniel Losen y Gary Orfield, eds., Racial Inequity in Special Education (Cambridge: Harvard Education Press, 2002); Gutiérrez-Saldivia, Quintriqueo y Valdebenito, "Carácter monocultural”; Ximena Gutiérrez-Saldivia y Enrique Riquelme, "Evaluación de necesidades educativas especiales en contextos de diversidad sociocultural: Opciones para una evaluación culturalmente pertinente", Revista Brasileira de Educação Especial vol. 26, n. ${ }^{\circ} 1$ (2020) doi: http://dx.doi.org/10.1590/s141365382620000100010; Ximena Gutiérrez-Saldivia, "Desproporcionalidad de estudiantes de grupos minoritarios en la educación especial", Revista Espacios vol. 39, n. ${ }^{\circ} 43$ (2018).

26 MINEDUC, Nueva perspectiva y visión de la Educación Especial. Informe de la Comisión de Expertos. (Santiago de Chile: Gobierno de Chile, 2004).

27 Fundación Chile, Informe final de estudio: "Análisis de la implementación de los Programas de Integración Escolar (PIE) en Establecimientos que han incorporado Estudiantes con Necesidades Educativas Especiales transitorias (NEET)" (Santiago de Chile: Centro de Innovación en Educación, 2013).

28 Consejo Nacional de la Cultura y las Artes, Región de La Araucanía, Síntesis Regional 2015 (Santiago de Chile: Departamento de Estudios, 2015); INE, Síntesis de resultados Censo 2017 (Santiago de Chile: Departamento de Estudios, 2018).

29 INE, Síntesis de resultados. 
sociales con orígenes históricos, cuyas tensiones se deben al proceso de colonización y posterior "pacificación de La Araucanía", momentos en los que se destituyó territorialmente al pueblo mapuche de sus tierras mediante sometimiento y procesos de aculturación ${ }^{30}$, para ello se recurrió principalmente a la castellanización, religión y escolarización de las personas ${ }^{31}$. Dichos procesos, no resueltos todavía, han decantado en relaciones culturales complejas entre el grupo dominante (chilenos) y dominados (mapuches), expresada mediante actos de racismo ${ }^{32}$ y discriminación, los que han permeado la sociedad en diversos ámbitos, incluida la educación ${ }^{33}$.

Finalmente, la metodología empleada en este trabajo corresponde a una revisión bibliográfica de carácter cualitativa-descriptiva, donde se analizaron evidencias de investigación empíricas y teóricas publicadas en revistas científicas indexadas y en libros sobre educación y Educación Especial. Esta última, considerada como modalidad educativa del sistema escolar regular, en contextos educativos de diversidad social y cultural. Para la recuperación de los documentos se utilizaron bases de datos.

\section{Educación Especial, modelo médico y diferencia}

La Educación Especial se consideró, desde sus inicios, como una educación paralela al sistema escolar regular, orientada al tratamiento y la rehabilitación de personas en situación de discapacidad ${ }^{34}$. Esto ha ido cambiando a través de los años, siendo definida en la actualidad como un subsistema o modalidad de la educación general, para dar respuestas educativas a estudiantes con $\mathrm{NEE}^{35}$. En Chile, la Educación Especial se basa en un enfoque de integración escolar dirigido a ofrecer apoyos educativos a estudiantes considerados especiales o con discapacidad en la educación regular. Este enfoque es definido como una estrategia comprensiva de la diversidad, donde se diseñan intervenciones educativas para atender las necesidades individuales de los estudiantes ${ }^{36}$; sin embargo, la integración no ha logrado impactar en los sistemas educativos ya que transfirió la Educación Especial arraigada en el modelo médico a las escuelas regulares, manteniendo una concepción negativa de las diferencias asociadas al déficit ${ }^{37}$. Así, al centrarse en el déficit, el modelo médico etiqueta a los estudiantes con

30 Donoso, Educación y nación.

31 José Bengoa, Mapuche, colonos y el Estado Nacional (Santiago de Chile: Catalonia, 2018).

32 María Pía Poblete, "Discriminacion étnica en relatos de la experiencia escolar mapuche en Panguipulli (Chile)", Estudios Pedagógicos. n. ${ }^{\circ} 29$ (2003). doi:https://doi.org/10.4067/S0718-07052003000100004.

33 Sandra Becerra, Consuelo Beldaño, Andrea Dastro y Jocelyn Coñuepan, "Prejuicio y discriminación étnica docente hacia niños indígenas en la escuela”, Teoria e Prática da Educação vol. 4, n. ${ }^{\circ} 1$ (2011). doi:https://doi.org/10.1016/j.sbspro.2015.07.107.

34 Erika González, "Evolución de la Educación Especial: del modelo del déficit al modelo de la Escuela Inclusiva”, en El largo camino hacia una educación inclusiva: La educación especial y social del siglo XIX a nuestros días: XV Coloquio de Historia de la Educación, coord. María Reyes y Susana Conejero (Pamplona: Universidad Pública de Navarra, 1. ${ }^{\circ}$ de julio de 2009).

35 Thomas Shea y Anne Marie Bauer, Educación especial: un enfoque ecológico (México, D.F.: McGraw-Hill, 2000).

36 Essomba, Liderar escuelas interculturales.

37 Mel Ainscow, "Haciendo que las escuelas sean más inclusivas: lecciones a partir del análisis de la investigación internacional", Revista de Educación Inclusiva vol. 5, n. ${ }^{\circ} 1$ (2012). 
discapacidades y trastornos ${ }^{38}$. La transferencia de este modelo a la educación proporciona poder a los actores educativos para buscar déficits en los estudiantes que no siguen el ritmo de aprendizaje esperado o que se comportan de manera diferente $^{39}$. Es así como mediante este modelo, en la escuela se diagnostica y se medicaliza la diferencia como discapacidad, lo que conlleva a estigmatizar, discriminar y marginar a los estudiantes que son derivados a la Educación Especial $^{40}$. La medicalización es entendida como "el proceso de convertir situaciones que han sido siempre normales en cuadros patológicos y pretender resolver, mediante la medicina, situaciones que no son médicas, sino sociales, profesionales o de las relaciones interpersonales" ${ }^{\prime \prime 1}$.

En este contexto, la Educación Especial al tener una base epistémica marcada por el modelo médico ${ }^{42}$, hace que los problemas de aprendizaje sean atribuibles a deficiencias o limitaciones personales y sociales ${ }^{43}$. Esto conlleva a que las instituciones escolares continúen centrando sus esfuerzos en buscar déficit en los estudiantes, más que en problematizar sus formas de enseñanza. Por ejemplo, el estudio de Núñez ${ }^{44}$ da cuenta que, en España, tres cuartas partes de los estudiantes con NEE que reciben apoyos educativos en centros de educación regular no sufren ninguna alteración orgánica. La autora, atribuye que el principal problema es que dichos estudiantes no pueden responder adecuadamente a las demandas del sistema educativo.

De esta forma, el modelo médico en educación, fomenta la etiquetación y clasificación de los estudiantes que poseen características peculiares con respecto al grupo mayoritario dominante. Así, desde este modelo se asumen las diferencias como deficiencias, debiendo identificar en los estudiantes rasgos de cada tipo de déficit según la nosología psiquiátrica de cada tiempo ${ }^{45}$. Esto justifica en educación la asignación de una determinada etiqueta a los estudiantes considerados diferentes para otorgar apoyos educativos ${ }^{46}$.

Una de las mayores críticas a la Educación Especial y al modelo médico, es ser el portavoz de una ideología de la normalidad. Esto, porque la discapacidad se instaló en la sociedad como una homologación al déficit, concepto anclado en la construcción de la idea de normalidad del cuerpo ${ }^{47}$. Así, desde la cultura

38 David Philpott, Assessing without labels: inclusive education in the Canadian context (Nunavut: Centre of Excellence for Children \& Adolescents with Special Needs, 2007).

39 María Julia Baltar, "El sentido del diagnóstico psicológico escolar. Un análisis crítico y una propuesta en construcción", Psicoperspectivas. Individuo y Sociedad vol. 2, n. ${ }^{\circ} 1(2003)$.

40 Ibíd.

41 Ramón Orueta Sánchez, Coral Santos Rodríguez, Enrique González Hidalgo, Eva Fagundo Becerra, Gemma Alejandre Lázaro, Javier Carmona de la Morena, Javier Rodríguez Alcalá, José María del Campo, María Luisa Díez Andrés, Natalia Vallés Fernández y Teresa Butrón Gómez, "Medicalización de la vida (I)", Revista Clínica Médica Familiar vol. 4, n. ${ }^{\circ}$ (2011): 150.

42 María Infante, "Desafíos a la formación docente: inclusión educativa”, Estudios Pedagógicos vol. 36, n. 1 (2010).

43 Mel Ainscow, Necesidades especiales en el aula. Guía para la formación del profesorado (Madrid: Narcea, 2001); Juan Escudero y Begoña Martínez, "Educación inclusiva y cambio escolar," Revista Iberoamericana de Educación, n. ${ }^{\circ} 5$ (2011).

44 María Núñez, “Educación especial: normalizar ou volver patolóxicas as diferenzas?”, Eduga n. 52 (2008).

45 González, "Evolución de la Educación Especial".

46 Alexis Soto Salcedo, "Desafíos de la psicología en el sur de Chile", en Análisis transdisciplinar de la salud en el sur de Chile (Soria: Editorial CEASGA, 2018).

47 María Alfonsina Angelino, "La discapacidad no existe, es una invención. De quienes fuimos (somos) siendo en el trabajo y la 
occidental las personas con discapacidad en el ámbito educativo, son etiquetas desde el concepto de NEE con el objetivo de reemplazar el lenguaje tradicional de carácter peyorativo vinculado a expresiones como enfermo, discapacitado, inválido o retrasado ${ }^{48}$.

Desde esta perspectiva, la Educación Especial tiene como función principal en la escuela medir, diagnosticar y clasificar a aquellos estudiantes que no se ajustan al tipo de sujeto que el sistema escolar busca formar. En palabras de Foucault $\mathrm{t}^{49}$ quien no se ajusta a esa normalidad, es decir de estudiante promedio, debe ser controlado, corregido o castigado por intervenciones específicas. En el sistema escolar esto se evidencia en la asignación de etiquetas diagnósticas y el ingreso de estos estudiantes a programas de apoyos de Educación Especial para ser corregidos.

En consecuencia, la etiquetación implica una lógica enjuiciadora que clasifica a los estudiantes según criterios de normalidad y anormalidad ${ }^{50}$, lo que en educación se aplica a través de test, que son estandarizados bajo el modelo de distribución normal, propio de la estadística. Cabe destacar que la noción de normalidad en educación subyace al concepto de "hombre medio" acuñado por Adolphe Quetelet en la primera mitad del siglo XIX, quien aplicó el modelo de curva normal a la medición de las características humanas ${ }^{51}$. Entonces, la aplicación de este modelo generó que se definieran normas estadísticas sobre las poblaciones ${ }^{52}$, valorando a las personas como normales o anormales según el intervalo donde se ubiquen ${ }^{53}$. Este intervalo se grafica en la denominada "Campana de Gauss", que determina umbrales de normalidad y desviaciones o extremos ${ }^{54}$.

En el caso de la educación, la normalidad se relaciona con la secuencia lineal de periodos del desarrollo (edades) que se corresponden con la secuencia del currículum escolar, tanto en contenidos como aprendizajes, creando un imaginario de progreso lineal-universal, exigible para todos ${ }^{55}$. Así, se construye una visión de normalidad con base en hitos que se establecen en el currículum y ritmos de desarrollo según la edad de los estudiantes, y "quienes se salgan del estándar normativo, quienes no sigan el ritmo y la secuencia, caen en la anorma-

producción", en Discapacidad e ideología de la normalidad. Desnaturalizar el déficit, eds. Ana Rosato y María Alfonsina Angelino (Buenos Aires: Noveduc, 2009).

48 Mónica Peña, "Análisis crítico de discurso del decreto 170 de subvención diferenciada para necesidades educativas especiales: el diagnóstico como herramienta de gestión", Psicoperspectivas vol. 12, n. ${ }^{\circ} 3$ (2013).

49 Michel Foucault, Vigilar y castigar: El nacimiento de la prisión (Buenos Aires: Siglo XXI Editores, 2009).

50 Julia Baltar, "El sentido del diagnóstico psicológico escolar. un análisis crítico y una propuesta en construcción”, Psicoperspectivas vol. 2, (2003).

51 Indiana Vallejos, "La categoría de normalidad: una mirada sobre viejas y nuevas formas de disciplinamiento social", en Discapacidad e ideología de la normalidad. Desnaturalizar el déficit, eds. Ana Rosato y María Alfonsina Angelino (Buenos Aires: Noveduc, 2009).

52 Gimeno Sacristán, "La construcción del discurso".

53 Vallejos, "La categoría de normalidad".

54 Yennifer Villa Rojas, "Hegemonía de la normalidad en la escuela”, Educación y Ciudad n. 33 (2009).

55 Gimeno Sacristán, "La construcción del discurso". 
lidad, bien sea en una zona negativa (retraso, subnormalidad...), bien en su zona positiva (adelanto, sobredotación)" 56 .

En contextos de diversidad social y cultural, el pensar las diferencias desde ideas de normalidad y anormalidad se considera una concepción colonial debido a que se focaliza en la medicalización y creación de diagnósticos mediante una perspectiva de desviación basada en una norma que se establece desde el grupo dominante a la población ${ }^{57}$. Esta concepción de diferencia puede provocar problemas cuando hay grupos sociales que poseen diversas normas o estándares, o diferentes formas de entender, por ejemplo, enfermedades y discapacidades ${ }^{58}$. En sociedades multiculturales es un problema identificar dificultades de aprendizaje o de conducta desde esta concepción de diferencia, ya que se asumen normas culturales homogéneas en lugar de normas heterogéneas o indígenas ${ }^{59}$.

En este contexto, la Educación Especial es funcional al modelo educativo homogeneizador, ya que otorga poder a los actores educativos para diagnosticar a estudiantes cuyos desempeños se alejan de la norma en términos de comportamiento o rendimiento. Lo descrito decanta en una concepción negativa de la diversidad, la cual considera que las habilidades de los estudiantes de la cultura dominante son normales y las variaciones sociales y culturales presentes en otros grupos, como es el caso de los estudiantes indígenas, son deficiencias ${ }^{60}$. Es así como la Educación Especial, a través de su sistema de etiquetado, denominado evaluación de NEE, hace visibles las diferencias en vez de "acoger al otro [...] sin importar su nombre, su lengua, su aprendizaje, su comportamiento, [su cultura y] nacionalidad"61. Así, las causas de la desviación recaen en la persona y no en la institución. Esta idea subyace a una concepción individual de las $\mathrm{NEE}^{62}$.

Según Ainscow ${ }^{63}$, la concepción individual de las NEE posee varias características principales. La primera se centra en identificar a un grupo de estudiantes como diferentes, dividiendo a la población escolar en dos grupos, los estudiantes que necesitan apoyos especiales de los que no los necesitan; la segunda característica se relaciona con establecer que solo un grupo de estudiantes requiere de apoyos especiales; la tercera resalta la idea que los problemas de los estudiantes considerados diferentes son el resultado de sus deficiencias, teniendo que ser diagnosticados y tratados; la cuarta característica tiene relación con la entrega de apoyos o ayudas especiales en grupos homogéneos, ya sea en escuelas espe-

\footnotetext{
56 Ibíd., 29.

57 Sarah Nelson, “Challenging Hidden Assumptions: Colonial Norms as Determinants of Aboriginal Mental Health”, National Collaborating Centre for Aboriginal Health, 2012.

58 Ibíd.

59 Janette Klingner, Alfredo Artiles, Elizabeth Kozleski, Beth Harry, Shelley Zion, William Tate, Grace Zamora Durán y David Riley, "Addressing the disproportionate representation of culturally and linguistically diverse students in special education through culturally responsive educational systems", Education Policy Analysis Archives vol. 13, n. 38 (2005).

60 Barbara Rogoff y Pablo Chavajay, "Las bases culturales del desarrollo cognitivo: evolución de la investigación en este campo en Norteamérica”, Educación y Pedagogía vol. 16, n. ${ }^{\circ} 39$ (2004).

61 Carlos Skliar, "Fragmentos de amorosidad y de alteridad en educación”, Revista Colombiana de Educación n. 50 (2006): 259.

62 Gillian Fulcher, Disabling Policies? A comparative approach to education policy and disability (Londres: Routledge, 1989).

63 Ainscow, Necesidades especiales en el aula.
} 
ciales, aulas especiales o programas de apoyos. Finalmente, la característica de la concepción individual de las NEE consiste en que se clasifica a los estudiantes en dos categorías, normales y diferentes, con base en una norma.

En síntesis, la Educación Especial ha aportado una mirada clínica sobre los estudiantes en general, y sobre los indígenas en particular, la cual insiste hasta la actualidad en patologizar la diversidad mediante el diagnóstico de discapacidades y trastornos, ocultándose en la escuela bajo el argumento de apoyos o recursos extraordinarios para estudiantes con aparentes barreras en el aprender y participar. Así, la escuela se sustenta "en un ideal de sujeto blanco, intelectual, hombre, burgués, católico y heterosexual, [donde] cualquier desviación implica una división punitiva $[\ldots]$ de los estudiantes [...]; [así] los 'defectuosos' [...], deben ser observados para encontrar el origen de su déficit" ${ }^{\prime \prime 4}$.

\section{Poder y teoría del etiquetado en la Educación Especial}

Autores como Apple señalan que "las instituciones de conservación y distribución de la cultura, como las escuelas, crean y recrean formas de conciencias que permiten el mantenimiento del control social sin que los grupos dominantes tengan necesidad de recurrir a mecanismos manifiestos de dominación" ${ }^{\prime 65}$. En este contexto, Apple expone que se deben analizar las concepciones que subyacen a las reformas educativas ya que, así como poseen argumentos asociados a crear condiciones más humanas, también tienen efectos más nocivos que útiles. Este es el caso de las políticas de educación que fomentan el etiquetado de los estudiantes para otorgar apoyos educativos desde programas de Educación Especial, y aunque se plantean como dispositivos de ayuda, son "mecanismos mediante los cuales la escuela se compromete a la anonimización y clasificación de los individuos abstractos en lotes sociales, económicos y educativos preordena$\operatorname{dos}^{\prime \prime 66}$. Autores como Schur ${ }^{67}$ manifiestan que el etiquetado funciona como una forma de control social para homogeneizar la realidad social y eliminar otras formas de pensamiento. El fenómeno de la etiquetación en educación requiere de una interpretación social crítica que indague en las relaciones de poder ${ }^{68}$.

Una perspectiva que permite explicar las prácticas de diagnóstico de NEE en la educación es la teoría de la etiquetación. Esta es una teoría micro sociológica desarrollada en Estados Unidos en la década de los 60, la cual postula que los grupos sociales son los que crean la desviación, donde establecen reglas, y su infracción constituye dicha desviación, y al aplicar estas reglas a determinadas personas, estas son etiquetadas como desviadas ${ }^{69}$. Así, desde esta teoría,

\footnotetext{
64 Villa, "Hegemonía de la normalidad", 122.

65 Michael W. Apple, Ideología y currículo (Madrid: Ediciones Akal, 1986), 13.

66 Ibíd., 166.

67 Edwin M. Schur, Labeling deviant behavior: Its sociological implications (Oxford: Harper \& Row, 1971).

68 Apple, Ideología y currículo.

69 Howard S. Becker, "Social-Class Variations in the Teacher-Pupil Relationship," The Journal of Educational Sociology vol. 25, n. 8 (1952).
} 
la desviación es entendida como una construcción social por parte del grupo mayoritario, que califica negativamente los comportamientos de personas pertenecientes a otros grupos sociales y culturales, y al no cumplir con las normas establecidas por la mayoría, se etiquetan como desviados ${ }^{70}$.

En palabras de Becker, la desviación se trata de un juicio social impuesto, es decir, creado por la sociedad ${ }^{71}$. Por lo tanto, no es producto de una acción, sino la consecuencia de aplicar reglas y sanciones ${ }^{72}$. En el contexto de la Educación Especial, son los actores educativos, como los profesores y profesionales de la educación, quienes deben identificar al estudiante desviado según las normas y reglas establecidas por las políticas educativas. Cabe destacar que la teoría del etiquetado tiene como objetivo demostrar que las fuerzas de control social frecuentemente tienen la consecuencia intencionada de definir a las personas como desviadas ${ }^{73}$. Los estudios basados en esta teoría centran su atención en los jueces de la desviación y las fuerzas que afectan sus juicios, donde emplean diversas fuentes de información ${ }^{74}$. Desde la idea anterior, en el contexto educativo, las fuentes de información que emplean los profesores u otros profesionales se basan en información primaria y secundaria. La información primaria es lo que surge de la interacción con los estudiantes (principalmente en el aula) y sus expectativas, mientras que la información secundaria es la que emerge desde otras fuentes, como por ejemplo los comentarios de otros profesores, los resultados de pruebas estandarizadas, resultados de informes anteriores, entre otros.

Cabe destacar que existen estudios que han demostrado que las variables raciales y étnicas son factores poderosos en la formulación de expectativas de los profesores y en su etiquetación ${ }^{75}$. Por ejemplo, el estudio realizado por Becker devela que los profesores que participaron en su estudio esperan menos de los niños de clase baja que de los de clase media ${ }^{76}$. En educación influyen en la etiquetación de los estudiantes variables como la raza, clase social, sexo, comportamiento aparente, edad y ocupación ${ }^{77}$.

Desde lo ya expuesto, la Educación Especial podría ser considerada un dispositivo de dominación y control social, que materializa el poder mediante un sistema de evaluación de NEE que emplea procedimientos de examen, pruebas o test estandarizados, para etiquetar, clasificar y diferenciar a la población que ha de ser enseñada. De acuerdo con Foucault ${ }^{78}$ los procedimientos de examen

70 Ray Rist, "Sobre la comprensión del proceso de escolarización: Aportaciones de la teoría del etiquetado", Educación y Sociedad n. 9 (1991).

71 Howard S. Becker, Outsiders. Studies in the sociology of deviance (Nueva York: The Free Press, 1968).

72 Rist, "Sobre la comprensión del proceso".

73 Ibíd.

74 Ibíd.

75 Bert Brown, The Assessment of Self Concept Among Four-Year-Old Negro and White Children: A Comparative Study Using the Brown-IDS Self Concept Referents Test (Nueva York: Institute for Developmental Studies, 1966); Gregg Jackson y Cecilia Cosca, "The Inequality of Educational Opportunity in the Southwest: An Observational Study of Ethnically Mixed Classrooms", American Educational Research Journal vol. 11, n. ${ }^{\circ} 3$ (1974).

76 Becker, "Social-Class Variations".

77 Rist, "Sobre la comprensión".

78 Foucault, Vigilar y castigar. 
son utilizados como una mirada documentada de carácter normalizador, ya que permiten clasificar, formar categorías, establecer medidas y fijar normas en la población, haciendo visibles las diferencias entre las personas. Foucault define el examen como un "sistema comparativo que permite la medición de fenómenos globales, la descripción de grupos, la caracterización de hechos colectivos, la estimación de las desviaciones de los individuos respecto de otros, y su distribución en una población"79. En la escuela, el examen es legitimado como una herramienta para identificar a estudiantes que presentan NEE, pues se considera un procedimiento objetivo que tiene un carácter científico ${ }^{80}$. En esta supuesta objetividad técnica "se encuentran implicados todo un dominio de saber, todo un tipo de poder" 81 aplicado al diagnóstico escolar. Foucault señala que "la superposición de las relaciones de poder y de las relaciones de saber adquieren en el examen toda su notoriedad visible ${ }^{\prime 82}$, lo que se materializa en las instituciones educativas mediante la administración de test de inteligencia y de rendimiento en los procesos de evaluación de NEE. Autores como Helms ${ }^{83}$ plantean que las pruebas de inteligencia incorporan valores implícitos que derivan de una perspectiva eurocéntrica, donde se aceptan los valores europeos como norma. En esta misma línea, Stairs y Bernhard ${ }^{84}$ señalan que los test son un artefacto de la cultura occidental que transmite la mentalidad colonial.

En este contexto, la Educación Especial actúa como un dispositivo para mantener la hegemonía en contextos de diversidad social y cultural. La hegemonía, concepto acuñado por Gramsci, es definida por McLaren como el "mantenimiento de la dominación no por el mero ejercicio de la fuerza, sino principalmente a través de prácticas sociales consensuales, formas sociales y estructuras sociales producidas en sitios específicos como la Iglesia, el Estado, la escuela, los medios de comunicación, el sistema político y la familia" ${ }^{85}$. En este contexto, en la Educación Especial la hegemonía se transmite a través de sus prácticas, en especial la evaluación de NEE, ya que se sustenta en el modelo médico que considera una única forma de ser y estar en el mundo, aplicando tan solo normas y estándares occidentales-universales, que excluye de su estructura la diversidad social y cultural. Esta práctica es en general aceptada por la comunidad educativa y las familias, pues su poder se encuentra oculto en las buenas intenciones de apoyar el aprendizaje de los estudiantes.

En contexto indígena, la Educación Especial como modalidad del sistema escolar mantiene y potencia una idea negativa de estos estudiantes, que han sido considerados históricamente como inferiores y menos inteligentes. Ejemplo

\footnotetext{
79 Ibíd., 221.

80 Gimeno Sacristán, "La construcción del discurso".

81 Foucault, Vigilar y castigar, 215.

82 Ibíd.

83 Janet Helms, "Why is There No Study of Cultural Equivalence in Standardized Cognitive Ability Testing?", American Psychologist vol. 47, n. ${ }^{\circ} 9$ (1992).

84 Arlene Stairs y Judith K. Bernhard, "Considerations for Evaluating 'Good Care' in Canadian Aboriginal Early Childhood Settings", McGill Journal of Education vol. 37, n. 3 (2002).

85 Peter McLaren, Life in schools. An Introduction to Critical Pedagogy in the Education (New York: Longman, 1989), 173.
} 
de esta visión sobre los niños y niñas indígenas se puede evidenciar en las ideas expuestas por Guevara ${ }^{86}$ en su libro Psicolojia del pueblo araucano publicado en 1908, donde se caracteriza a la población indígena mapuche como incivilizada y deficiente intelectualmente. Esto se puede explicar desde el punto de vista de la sociología de las ausencias, entendida como aquellas investigaciones que tienen como objetivo mostrar lo que no existe. Santos ${ }^{87}$ describe cinco formas distintas de producción de no existencia, las que están unidas por la racionalidad monocultural. Una de estas formas de reproducción, vinculadas a la Educación Especial y más específicamente al diagnóstico, es la lógica de la clasificación social, vinculada a la monocultura de la naturalización de las diferencias. La lógica de clasificación social "consiste en la distribución de las poblaciones por categorías que naturalizan jerarquías" 88 . Desde esta perspectiva pensar en estudiantes con NEE y otros sin ella da poder a los actores sociales para considerar a unos como grupo enfermo-anormal y a otros como sano-normal, justificando el uso de etiquetas y apoyos educativos extraordinarios en las escuelas.

En suma, tanto la perspectiva de poder y la teoría de la etiquetación aportan a la comprensión de la Educación Especial en contextos indígenas. El punto común de ambas perspectivas es que, una vez que se diagnostica al estudiante, se debe responder institucionalmente a la diferencia mediante la corrección y tratamiento de esta, centrando la atención en la persona.

\section{CONCLUSIONES}

El análisis desarrollado en este trabajo nos permite sostener que el sistema escolar fomenta una visión de la diversidad como déficit, anormalidad y desviación, desde la modalidad de Educación Especial. Esta perspectiva de la Educación Especial en contextos de diversidad social y cultural podría ser considerada como un dispositivo de poder que busca la homogenización de todo aquel que no se asemeja a la cultura dominante. Para esto utiliza como herramienta el diagnóstico de NEE mediante el uso de exámenes o test estandarizados que legitiman la etiquetación y clasificación de los estudiantes en la escuela. Así, en el contexto de América Latina, las prácticas asociadas a la Educación Especial se relacionan con el imaginario de indígenas desposeídos del conocimiento eurocéntrico-occidental, lo cual los margina de las exigencias de las estructuras del saber y del poder de la sociedad colonizada ${ }^{89}$. Según lo descrito, los sistemas educativos han tenido la función de transmitir conocimientos eurocéntrico-occidentales como únicos y verdaderos

86 Tomás Guevara, Psicolojía del pueblo araucano (Santiago de Chile: Cervantes, 1908).

87 Boaventura de Sousa Santos, Descolonizar el saber, reinventar el poder (Montevideo: Ediciones: Trilce, 2010).

88 Ibíd., 23.

89 Aníbal Quijano, “Colonialidad del poder, eurocentrismo y América Latina”, en La colonialidad del saber: eurocentrismo y ciencias sociales. Perspectivas latinoamericanas, ed. Edgardo Lander (Buenos Aires: Concejo Latinoamericano de Ciencias Sociales, 2000). 
para formar a las personas, omitiéndose la matriz social y cultural de los estudiantes indígenas ${ }^{90}$, y quien no adquiere los valores y conocimientos de la cultura dominante debe ser tratado y corregido por una pedagogía especializada, como la Educación Especial.

En respuesta a lo anterior, los contextos de diversidad social y cultural requieren una nueva forma de pensar y orientar la Educación Especial para romper con la estructura y cánones occidentales arraigados en las formas de concebir las cuestiones asociadas a la diversidad y las NEE. Para lograr lo anterior, se hace necesario repensar la Educación Especial desde la interculturalidad, y así cuestionar la perspectiva monocultural-universalista que ha predominado en la escuela occidental.

Finalmente, a partir de lo expuesto en este trabajo se concluyen los siguientes puntos:

1) Se requiere re-pensar la Educación Especial de forma urgente a través de un análisis crítico, que reconozca su episteme y limitaciones en contextos educativos interculturales, como es el caso de la región de La Araucanía.

2) Una posibilidad para re-pensar la Educación Especial se basa en la perspectiva intercultural, lo cual implicaría la apertura de los actores educativos al diálogo con "el otro", desde la valoración y el respeto, favoreciendo intervenciones educativas con pertinencia cultural, donde las diferencias no sean consideradas como NEE.

3) Se propone eliminar las prácticas de diagnóstico y etiquetación, con el objetivo de avanzar en la erradicación de aquellas representaciones de las diferencias asociadas al déficit.

4) Es necesario que los agentes educativos, especialmente profesores y profesionales de apoyo de las escuelas, adopten una postura crítica y autónoma en la toma de decisiones en los procesos de evaluación e intervención psicoeducativa.

5) Es imprescindible que la Formación Inicial Docente, y carreras profesionales afines a la educación, desarrollen una perspectiva crítica frente al concepto de déficit y necesidades educativas especiales, con el propósito de erradicar la visión homogeneizadora y universalista que domina epistemológicamente los saberes pedagógicos y disciplinares.

\section{REFERENCIAS}

Ainscow, Mel. “Haciendo que las escuelas sean más inclusivas: lecciones a partir del análisis

90 Daniel Quilaqueo, Segundo Quintriqueo y Héctor Torres, “Características epistémicas de los métodos educativos mapuche”, REDIE vol. 18, n. $^{\circ} 1$ (2016). 
de la investigación internacional". Revista de Educación Inclusiva 5, n. ${ }^{\circ} 1$ (2012): 39-41.

Ainscow, Mel. Necesidades especiales en el aula. Guía para la formación del profesorado. Madrid: Narcea, 2001.

Angelino, María Alfonsina. “La discapacidad no existe, es una invención. De quienes fuimos (somos) siendo en el trabajo y la producción". En Discapacidad e ideología de la normalidad. Desnaturalizar el déficit, editado por Ana Rosato y María Alfonsina Angelino. Buenos Aires: Noveduc, 2009.

Apple, Michael W. Ideología y currículo. Madrid: Ediciones Akal, 1986.

Assaél, Jenny, Natalia Albornoz y Miguel Caro. “Estandarización educativa en Chile: Tensiones y consecuencias para el trabajo docente". Educação Unisinos 22, n. ${ }^{\circ} 1$ (2018): 83-90.

Baltar, María Julia. “El sentido del diagnóstico psicológico escolar. Un análisis crítico y una propuesta en construcción". Psicoperspectivas. Individuo y Sociedad 2, n. ${ }^{\circ} 1$ (2003): 7-34.

Becerra, Sandra, Consuelo Beldaño, Andrea Dastro y Jocelyn Coñuepan. "Prejuicio y discriminación étnica docente hacia niños indígenas en la escuela". Teoria e Prática da Educação 4, n. ${ }^{\circ} 1$ (2011): 7-17.

Becker, Howard S. Outsiders. Studies in the sociology of deviance. Nueva York: The Free Press, 1968.

Becker, Howard. "Social-Class Variations in the Teacher-Pupil Relationship". The Journal of Educational Sociology 25, n. ${ }^{\circ} 8$ (1952): 451-465.

Bengoa, José. Mapuche, colonos y el Estado Nacional. Santiago de Chile: Catalonia, 2018.

Besalú, Xavier. Diversidad cultural y educación. Madrid: Síntesis Educación, 2002.

Brown, Bert. The Assessment of Self Concept Among Four-Year-Old Negro and White Children: A Comparative Study Using the Brown-IDS Self Concept Referents Test. Nueva York: Institute for Developmental Studies, 1966.

Fundación Chile. Informe final de estudio: "Análisis de la implementación de los Programas de Integración Escolar (PIE) en Establecimientos que han incorporado Estudiantes con Necesidades Educativas Especiales Transitorias (NEET)". Santiago de Chile: Centro de Innovación en Educación, 2013.

Consejo Nacional de la Cultura y las Artes. Región de La Araucanía. Síntesis regional (Santiago de Chile: Departamento de Estudios, 2015).

Donoso, Andrés. Educación y nación al sur de la frontera. Organizaciones mapuche en el umbral de nuestra contemporaneidad, 1880-1930. Santiago de Chile: Pehuén Editores S.A, 2012.

Donovan, Suzanne y Christopher Cross, eds. Minority Students in Special and Gifted Education. Washington, D.C.: The National Academies Press, 2002.

Escudero, Juan y Begoña Martínez. "Educación inclusiva y cambio escolar". Revista Iberoamericana de Educación n. ${ }^{\circ} 55$ (2011): 85-105.

Essomba, Miquel Àngel. Liderar escuelas interculturales e inclusivas. equipos directivos y profesorado ante la diversidad cultural y la inmigración. Barcelona: Graó, 2006.

Fernández, Francisco, "El estudio sociológico de la educación". En Sociología de la educación, coordinado por Francisco Fernández. Madrid: Pearson Prentice Hall, 2003.

Foucault, Michel. Vigilar y castigar: el nacimiento de la prisión. Buenos Aires: Siglo XXI Editores, 2009.

Fulcher, Gillian. Disabling Policies? A comparative approach to education policy and disability. Londres: Routledge, 1989.

Godoy, M. ${ }^{a}$ Paulina, M. ${ }^{a}$ Luisa Mezay Alida Salazar. Antecedentes históricos, presente y futuro de la Educación Especial en Chile (Santiago de Chile: Ministerio de Educación, 2004).

González, Erika. “Evolución de la Educación Especial: del modelo del déficit al modelo de la Escuela Inclusiva. En El largo camino hacia una educación inclusiva: la educación especial y social del siglo XIX a nuestros días: XV Coloquio de Historia de la Educación, coordinado por María Reyes y Susana Conejero. Pamplona: Universidad Pública de Navarra, 2009.

Guerrero, Antonio. Enseñanza y sociedad. El conocimiento sociológico de la educación. Madrid: Siglo XXI, 2005. 
Gutiérrez-Saldivia, Ximena y Enrique Riquelme. "Evaluación de necesidades educativas especiales en contextos de diversidad sociocultural: Opciones para una evaluación culturalmente pertinente". Revista Brasileira de Educação Especial 26, n. ${ }^{\circ} 1$ (2020): 159-174.

Gutiérrez-Saldivia, Ximena, Segundo Quintriqueo Millán y Vanessa Valdebenito Zambrano. "Carácter monocultural de la evaluación diagnóstica de necesidades educativas especiales en contexto mapuche". Educaçao e Pesquisa 45 (2019): 1-18.

Gutiérrez-Saldivia, Ximena. "Desproporcionalidad de estudiantes de grupos minoritarios en la educación especial". Revista Espacios 39, n. ${ }^{\circ} 43$ (2018): 1- 14.

Guevara, Tomás. Psicolojia del pueblo araucano. Santiago de Chile: Cervantes, 1908.

Helms, Janet. "Why Is There No Study of Cultural Equivalence in Standardized Cognitive Ability Testing?". American Psychologist 47, n. 9 (1992): 1083-1101.

INE (Instituto Nacional de Estadística de Chile). "Síntesis de resultados censo 2017" (Santiago de Chile: INE, 2018).

Infante, María. "Desafíos a la formación docente: inclusión educativa". Estudios Pedagógicos 36, n. ${ }^{\circ}$ (2010): 287-297.

Jackson, Gregg y Cecilia Cosca. "The Inequality of Educational Opportunity in the Southwest: An Observational Study of Ethnically Mixed Classrooms". American Educational Research Journal 11, n. ${ }^{\circ} 3$ (1974): 219-229.

Klingner, Janette, Alfredo Artiles, Elizabeth Kozleski, Beth Harry, Shelley Zion, William Tate, Grace Zamora Durán y David Riley. "Addressing the disproportionate representation of culturally and linguistically diverse students in special education through culturally responsive educational systems". Education Policy Analysis Archives 13, n. 38 (2005): 1-43.

Losen, Daniel y Gary Orfield, eds. Racial Inequity in Special Education. Cambridge: Harvard Education Press, 2002.

Magendzo, Abraham. "Alteridad y diversidad: componentes para la educación social". Pensamiento Educativo 37 (2005): 106-116.

McLaren, Peter. Life in Schools. An Introduction to Critical Pedagogy in the Education. New York: Longman, 1989.

Ministerio de Educación (MINEDUC). Ley Núm. 20.370 que establece la Ley General de Educación (Biblioteca del Congreso Nacional de Chile, 12 de septiembre de 2009).

Ministerio de Educación. Nueva perspectiva y visión de la Educación Especial. Informe de la Comisión de Expertos (Santiago de Chile: Gobierno de Chile, 2004).

Ministerio de Educación. "Orientaciones técnicas para Programas de Integración Escolar (PIE)". (Santiago de Chile: MINEDUC, 2013)

Nelson, Sarah. "Challenging Hidden Assumptions: Colonial Norms as Determinants of Aboriginal Mental Health". National Collaborating Centre for Aboriginal Health, 2012.

Núñez Mayán, María Teresa. “Educación especial: Normalizar ou volver patolóxicas as diferenzas?". Eduga n. ${ }^{\circ} 52$ (2008), 20-23.

Ogbu, John. "Cultural problems in minority education: Their interpretations and consequences-Part one: Theoretical backgroud". The Urban Review 27, n. 3 (1995): 189-205.

Oliva, María. "Política educativa chilena 1965-2009. ¿Qué oculta esa trama?". Revista Brasileira de Educação 15, n. ${ }^{\circ} 44$ (2010): 311-328.

Ornelas, Carlos. Educación y sociedad: ¿Consenso o conflicto?. En Sociología de la educación, editado por Carlos Alberto Torres y Guillermo González Rivera. Buenos Aires: Miño y Dávila editores, 1994.

Orueta Sánchez, Ramón, Coral Santos Rodríguez, Enrique González Hidalgo, Eva Fagundo Becerra, Gemma Alejandre Lázaro, Javier Carmona de la Morena, Javier Rodríguez Alcalá, José María del Campo, María Luisa Díez Andrés, Natalia Vallés Fernández y Teresa Butrón Gómez. "Medicalización de la vida (I)". Revista Clínica Médica Familiar 4, n. ${ }^{\circ} 2$ (2011): 150-161.

Peña, Mónica. "Análisis crítico de discurso del decreto 170 de subvención diferenciada para 
necesidades educativas especiales: el diagnóstico como herramienta de gestión". Psicoperspectivas 12, n. ${ }^{\circ} 3$ (2013): 93-103.

Philpott, David. Assessing without labels: Inclusive education in the Canadian context. Nunavut: Centre of Excellence for Children \& Adolescents with Special Needs, 2007.

Poblete, María Pía. "Discriminación étnica en relatos de la experiencia escolar mapuche en Panguipulli (Chile)". Estudios Pedagógicos n. ${ }^{\circ} 29$ (2003): 55-64.

Quijano, Aníbal. “Colonialidad del poder, eurocentrismo y América Latina.” En La Colonialidad del saber: eurocentrismo y ciencias sociales. Perspectivas Latinoamericanas, editado por Edgardo Lander. Buenos Aires: Concejo Latinoamericano de Ciencias Sociales, 2000.

Quilaqueo, Daniel, Segundo Quintriqueo y Héctor Torres. “Características epistémicas de los métodos educativos mapuche". REDIE 18, n. ${ }^{\circ} 1$ (2016): 153-165.

Redondo, Jesús. "El experimento chileno en educación: ¿Conduce a mayor equidad y calidad en la educación?". Última Década 13, n. ${ }^{\circ} 22$ (2005): 95-110.

Rist, Ray. "Sobre la comprensión del proceso de escolarización: aportaciones de la teoría del etiquetado". Educación y Sociedad n. ${ }^{\circ}$ (1991): 179-193.

Rogoff, Barbara y Pablo Chavajay. "Las bases culturales del desarrollo cognitivo: evolución de la investigación en este campo en Norteamérica". Educación y Pedagogía 16, n. 39 (2004): 121-159.

Gimeno Sacristán, José. "La construcción del discurso acerca de la diversidad y sus prácticas". En Atención a la diversidad. Barcelona: Graó, 2000.

Santos, Boaventura de Sousa. Descolonizar el saber, reinventar el poder. Montevideo: Ediciones Trilce, 2010.

Schur, Edwin M. Labeling deviant behavior: Its sociological implications. Oxford: Harper \& Row, 1971.

Shea, Thomas y Anne Marie Bauer. Educación especial: un enfoque ecológico. México, D.F.: McGraw-Hill, 2000.

Skliar, Carlos. "Fragmentos de amorosidad y de alteridad en educación". Revista Colombiana de Educación n. ${ }^{\circ} 50$ (2006): 253-266.

Soto Salcedo, Alexis. "Desafíos de la psicología en el sur de Chile". En Análisis transdisciplinar de la salud en el sur de Chile. Soria: Editorial CEASGA, 2018.

Stairs, Arlene y Judith K. Bernhard. "Considerations for Evaluating Good Care in Canadian Aboriginal Early Childhood Settings". McGill Journal of Education 37, n. ${ }^{\circ} 3$ (2002): 309-330.

Vallejos, Indiana. "La categoría de normalidad: una mirada sobre viejas y nuevas formas de disciplinamiento social". En Discapacidad e ideología de la normalidad. Desnaturalizar el déficit, editado por Ana Rosato y María Alfonsina Angelino. Buenos Aires: Noveduc, 2009.

Villa Rojas, Yennifer. "Hegemonía de la normalidad en la escuela". Educación y Ciudad n. 33 (2017): 115-126. 


\section{Cómo citar:}

Gutiérrez-Saldivia, Ximena; Rivera Gutiérrez, Catalina.

"Educación Especial y sus implicancias en contextos de diversidad cultural: análisis desde La Araucanía". Revista Historia de la Educación Latinoamericana. vol. 22 No. 34 (2020): 95-112

DOI: https://doi.org/10.19053/01227238.10106

(c) (i) @९ Esta obra está bajo una licencia Creative Commons. Reconocimiento-No Comercial-Sin Obra Derivada 2.5 Colombia. 